\title{
Clinical Outcomes and Prognostic Factors of Salvage Stereotactic Body Radiotherapy for Post-Surgical Thoracic Oligo-Recurrence/ Metastasis of Non-Small-Cell Lung Cancer
}

This article was published in the following Dove Press journal:

Cancer Management and Research

\author{
Wen-Cai Li ${ }^{1, *}$ \\ Zhen Wang ${ }^{2, *}$ \\ Jie $\mathrm{Gao}^{2}$ \\ Han Zhou ${ }^{2}$ \\ Jing $\mathrm{Li}^{2}$ \\ Xi-Xu Zhu (D) ${ }^{3}$ \\ 'Department of Radiation Oncology, \\ Jinling Hospital, The First School of \\ Clinical Medicine, Southern Medical \\ University, Guangzhou, People's Republic \\ of China; ${ }^{2}$ Department of Medical \\ Radiation Oncology, Jinling Hospital, \\ Nanjing, Jiangsu, 210002, People's \\ Republic of China; ${ }^{3}$ Department of \\ Medical Radiation Oncology, Jinling \\ Hospital, First School of Clinical \\ Medicine, Southern Medical University, \\ Nanjing, Jiangsu, 210002, People's \\ Republic of China
}

*These authors contributed equally to this work

\begin{abstract}
Purpose: The study aimed to assess the efficacy and safety of stereotactic body radiotherapy (SBRT) using CyberKnife (CK) in patients with postoperative thoracic oligo-recurrence/metastasis of non-small-cell lung cancer (NLCLC), and to analyze the prognostic factors affecting overall survival after SBRT.

Patients and Methods: A total of 44 patients with postoperative thoracic oligorecurrence/metastatic of NLCLC treated with SBRT were reviewed. Thoracic oligorecurrence/was defined as 1-3 loco-regional confined to lung lobe, hilar/mediastinal lymph nodes, bronchial stump, or chest wall. Primary endpoints included local control (LC), overall survival (OS), progression-free survival (PFS) and toxicity. Prognostic factors that affected these patients were analyzed by the univariate and multivariate analysis by Kaplan-Meier methods and Cox regression models, respectively.

Results: The median follow-up time after salvage SBRT was 48.5 months. Measuring from the date of salvage SBRT, the median OS of the 44 patients was 52.60 (95\% CI: 29.5975.60) months. 1-,3-and 5-year OS rates were $97.7 \%, 65.3 \%$ and $47.7 \%$, respectively. The 1 -,3-year and 5 -year LC rates were $97.7 \%, 85.1 \%$ and $80.1 \%$, respectively. At 1,3 and 5 years, the PFS rates were $77.1 \%, 28.8 \%$ and $5.3 \%$, respectively. Multivariate analysis demonstrated that pre-SBRT neutrophil-to-lymphocyte ratio (NLR) and Charlson comorbidity index $(\mathrm{CCI})$ were independent prognostic factors $(\mathrm{p}<0.05)$. The treatment-related sideeffects were well tolerated. No patients developed grade 3 or greater pulmonary toxicity.

Conclusion: SBRT is a promising salvage therapeutic option for postoperative thoracic oligo-recurrence/metastasis of non-small-cell lung cancer with acceptable toxicity. Low preSBRT neutrophil-to-lymphocyte ratio (NLR) and low Charlson comorbidity index (CCI) were associated with a better prognosis and longer survival and might be considered as reliable and independent prognostic factors in these patients treated with SBRT.
\end{abstract}

Keywords: oligo-recurrence, NSCLC, post-surgical recurrence, CyberKnife

\section{Introduction}

Complete surgical resection is the standard and recommended treatment for non-small cell lung cancer (NSCLC); however, post-surgical recurrence develops in about 30-55\% of patients with NSCLC after resection. ${ }^{1,2}$ It has been reported that postoperative NSCLC recurrence is associated with a poor prognosis. ${ }^{3,4}$ Recently, certain patients with a limited number of recurrent lesions, a state is called oligo-recurrence/metastasis, are expected to
Correspondence: Xi-Xu Zhu Department of Medical Radiation Oncology, Jinling Hospital, First School of Clinical Medicine, Southern Medical University, Nanjing, Jiangsu, 210002

People's Republic of China

Tel/Fax +86-025-80860078

Email zhuxixu@hotmail.com 
achieve long-term survival and even cure by definitive local therapy alone..$^{5-8}$ Although there is currently no clear consensus on the most suitable treatment, surgery is basically considered to be the primary choice, however, re-excision of the thoracic oligo-recurrence/metastasis lesions has been difficult due to anatomical structure change, poor baseline pulmonary function, high incidence of complications, and the low willingness of patients to undergo surgery again. ${ }^{9,10}$ For these reasons, less invasive therapies with limited risk of morbidity are often ideal. As a relatively minimally invasive definitive treatment approach, stereotactic body radiotherapy (SBRT) or stereotactic ablative body radiation (SABR) may be better suited to these patients, which uses advanced imaging and localizing techniques to optimize radiotherapy targeting accuracy which facilitates the delivery of hypo-fractionated, ablative doses of radiation ${ }^{11}$. SBRT or SABR has been proved to be an effective and tolerable treatment modality for both primary and metastatic lung cancer in many studies. ${ }^{12,13}$ However, there are few articles have reported the efficacy and safety of SBRT on post-operative thoracic oligorecurrence/metastasis of NSCLC. ${ }^{9}$

Therefore, our study aimed to retrospectively evaluate the efficacy and safety of stereotactic body radiotherapy in patients with postoperative thoracic oligo-recurrence /metastasis of Non-Small-Cell Lung Cancer, and to analyze the prognostic factors affecting overall survival after SBRT.

\section{Patients and Methods Clinical Data}

From September 2010 to July 2019, 44 patients with thoracic oligo-recurrence/metastasis were included in a retrospective study at the CyberKnife SBRT Center, Jinling Hospital. Prior to treatment, all patients underwent comprehensive staging, including head magnetic resonance imaging and 18 fluorine fluorodeoxyglucosepositron emission tomography/computed tomography (FDG-PET/CT), tumor markers, chest/abdomen enhanced computed tomography (CT), whole-body bone scanning, routine blood tests, and blood chemistry panels. The study protocol was in accordance with the ethical guidelines of the Declaration of Helsinki, and was approved by independent ethics committees in Jinling Hospital. In addition, all included patients provided a written informed consent.

Inclusion criteria: in this study, thoracic oligorecurrence/metastasis was defined as 1-3 loco-regional lesions confined to lung lobe, hilar/mediastinal lymph nodes, bronchial stump, or chest wall. We excluded patients if they: 1) were diagnosed as second primary lung cancers by experienced pathologists or thoracic oligorecurrence/metastasis but histologically unproved; 2) underwent initial resection of NSCLC without curative intent; 3) follow-up time $<6$ months or deficiency of follow-up information; 4) previous thoracic radiation therapy. This CONSORT diagram as is shown in Figure 1.

The following were collected and recorded from the electronic medical records system: the gender, age, smoking status, primary-tumor characteristics, disease free interval (the time interval between surgery and recurrence), Eastern Cooperation Oncology Group (ECOG) score, postoperative pathological staging (Staging of the primary tumor was conducted in accordance with the 8th edition of the Union for International Cancer Control staging system for lung cancer), neutrophil-to-lymphocyte ratio, platelet-to-lymphocyte ratio (PLR), hemoglobin levels comorbidities, Charlson comorbidity index, Epidermal growth factor receptor (EGFR) mutation status, median biological effective dose (BED10) levels, coverage, conformity index (CI), new conformity index (nCI), homogeneity index (HI) and the presence or absence of any treatments after SBRT.

\section{Positioning and Target Delineation}

All 44 patients were treated using a CyberKnife SBRT system (Accuracy Incorporated, Sunnyvale, CA, USA) via respiration synchronous tracking (Synchrony), which was previously described by our institution. ${ }^{14}$ The patient took the supine position and was fixed with a vacuum pad. Spiral CT (Brilliance TM Big Bore, Philips, Netherlands) scanning was conducted with a slice thickness of $1 \mathrm{~mm}$. The scanning range was $15 \mathrm{~cm}$ above and below the lesion. The gross tumor volume (GTV) was defined as the tumor volume which was delineated on the lung window of the CT. The planning target volume (PTV) was obtained by expanding the GTV by $3 \mathrm{~mm}$ uniformly in all directions. The dose was prescribed based on the isodose line and covered the PTV. SBRT was delivered to a total dose of 30 to 60 Gy over 2 to 6 fractions. The dose equivalence was used as a linear quadratic model and considered by assuming $\alpha / \beta=10$ Gy for the tumor. The biological effective dose (BED) ranged from 60-180 Gy, and the median $\mathrm{BED}_{10}$ was $124.8 \mathrm{~Gy}$. Dose and fractionation schedules were developed based on the tumor size, location of the lesions, and performance status. 


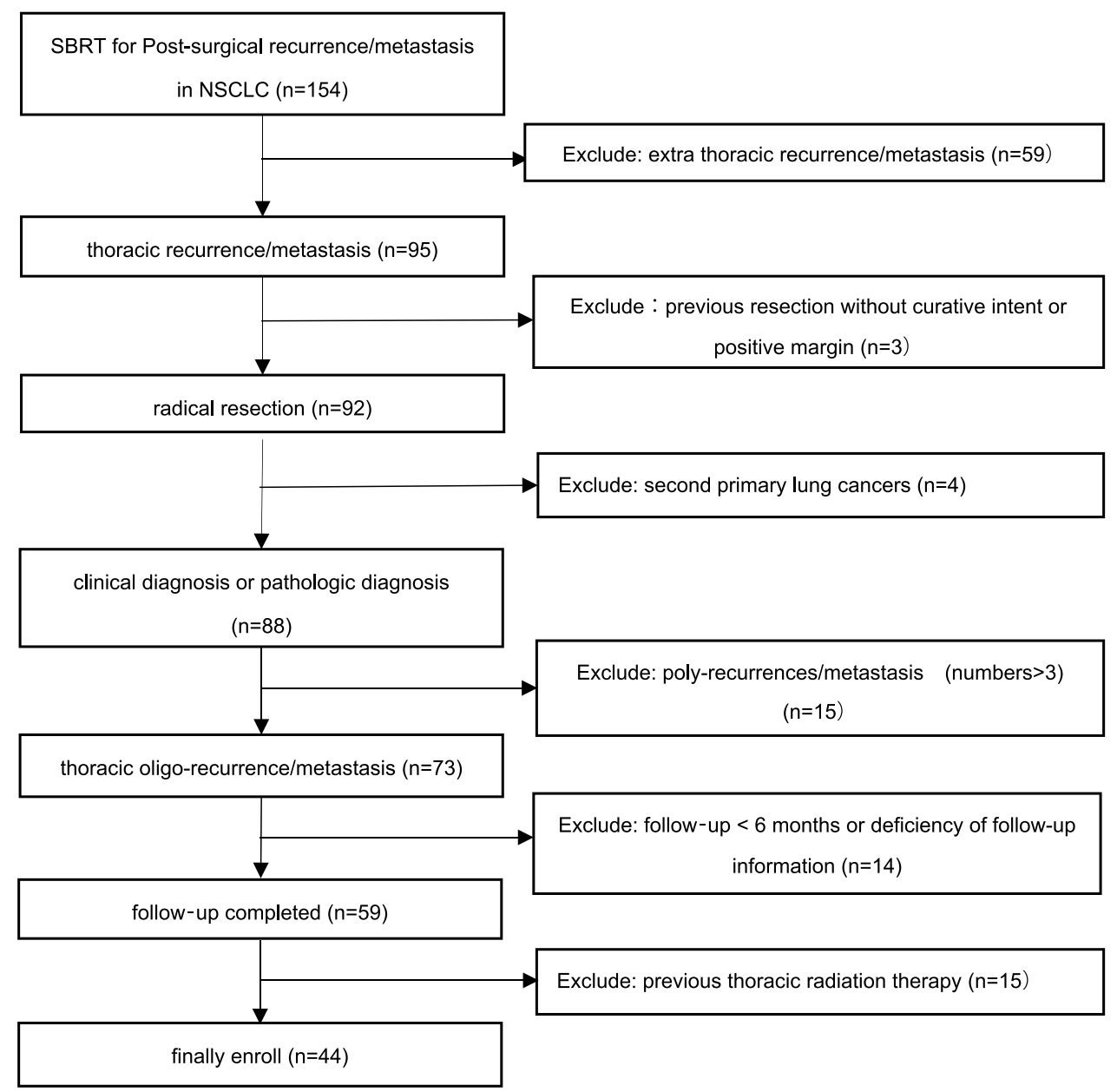

Figure I The CONSORT diagram for the study.

\section{Follow-Up and Adverse Reactions}

Follow-up started on the first day of radiotherapy initiation and ended on July 15, 2019. All patients underwent clinical examination and CT/PET-CT scan for evaluation of treatment effects 4-6 weeks after SBRT, then every 3 months for the first 2 years, and then the every 6-8 months until death or last follow up. Toxicity was assessed according to the Common Terminology Criteria for Adverse Events version 4.0. The Response Evaluation Criteria in Solid Tumors 1.1 (RECIST) was used to evaluate the treatment responses. Local recurrence after salvage SBRT was defined as in-field or marginal re-growth of the lesions. Regional recurrence was defined as the new appearance of positive mediastinal or hilar lymph nodes or the ipsilateral lung lesion. Distant metastasis was defined as metastasis to the contralateral lung and to outside of the hemithorax or mediastinum.

Overall survival (OS) was defined as the time from the first day of SBRT initiation to the date of mortality from any cause, or to the last follow-up visit or telephone contact prior to July 15, 2019. Progression-free survival (PFS) and local control (LC) was defined as the interval from the first day of SBRT to documented disease progression and locoregional recurrence, respectively. If SBRT was performed twice or more to treat post-surgical recurrent lesions after the first SBRT, PFS was defined as the interval between the first SBRT completion and disease progression.

\section{Statistical Analysis}

The SPSS 25.0 software was used for the statistical analysis of the research data. X-tile software was constructed to define the optimal cut-off values of the NLR and PLR. ${ }^{15}$ The LC, OS, and PFS rate estimates were calculated using the Kaplan-Meier method, and compared using the stratified Log rank test. The predictors of LC and OS after salvage SBRT were analyzed by using Kaplan-Meier methods and Cox regression models. $\mathrm{P}<0.05$ was considered statistically significant. 


\section{Results}

\section{Patient Characteristics}

The study population finally comprised 44 patients, with median age of 68 years (range, $30-80$ years). Pathological diagnosis at primary surgery was adenocarcinoma in 28/44 (63.6\%) patients and squamous carcinoma in 16/44 (36.4\%) patients. The originally treated tumor was stage I in 29 patients (65.9\%), stage II in 8 patients (18.2\%), and stage IIIA in $7(15.9 \%)$. Further, $9 / 44$ patients $(20.5 \%)$ exhibited pathological lymph node metastases at primary surgery. The median time from operation to recurrence was 22.43 (range,3.93-161.3) months, and the median time from relapse to $\mathrm{CK}$ was 1.87 (range,0.1-74.23) months. The median time from surgery to CK was 23.53 (range,4.17-171.6) months. The patient and tumor characteristics are detailed in Table 1.

\section{Dosimetric Index}

The isodose level of the prescription dose in the treatment plan was $66-89 \%$, with a median value of $80 \%$. The treatment plan revealed that the median $\mathrm{CI}, \mathrm{nCI}$, and homogeneity index (HI) of the recurrent lesions in all patients was $1.18,1.28$, and 1.24 respectively (Table 2).

\section{Treatment Outcomes}

For the 44 patients with postoperative thoracic oligorecurrence, the median follow-up duration after the start of SBRT was 48.5 months. The median OS of the 44 patients was 52.6 months (95\% CI 29.59-75.60 months). The 1-,3-year and, 5-year local control rates from the start of SBRT were $97.7 \%, 85.1 \%$, and $80.1 \%$, respectively (Figure 2A). The median PFS was 21.7 months (95\% CI $11.85-$ 31.55 months). At 1,3 , and 5 years, the disease-free survival rates were $77.1 \%, 28.8 \%$, and $5.3 \%$, respectively (Figure 2B). The 1-,3-and 5-year OS rates from the start of SBRT were $97.7 \%, 65.3 \%$, and $47.7 \%$ (Figure 2C), respectively.

\section{Prognostic and Predictive Factors of Local Control and Overall Survival}

Based on the X-TILE analysis, the optimal cut-off values of the NLR and PLR were 2.68 and 117.66, respectively. There was no statistical significance $(\mathrm{p}>0.05)$ in LC according to Univariate analysis (Table 3). Univariate analysis showed that several clinical factors, including ECOG performance status, tumor diameter, the pre-SBRT neutrophil-to-lymphocyte ratio (NLR), and Charlson
Table I CyberKnife Radiosurgery Treatment

\begin{tabular}{|c|c|}
\hline Characteristics & No. (\%) \\
\hline Age(years) at CK: median (range) & 68 years (39-85 years) \\
\hline \multicolumn{2}{|l|}{ Gender } \\
\hline Male & $35(79.5 \%)$ \\
\hline Female & 9 (20.5\%) \\
\hline \multicolumn{2}{|l|}{ Smoking status } \\
\hline Past smoker and current smoker & $22(50 \%)$ \\
\hline Never smoker & $22(50 \%)$ \\
\hline Disease free interval Median (range) & $\begin{array}{l}22.43 \text { months } \\
\text { (3.93-161.3 months) }\end{array}$ \\
\hline Symptoms at recurrence (yes/no) & $8(18.2 \%): 36(81.8 \%)$ \\
\hline \multicolumn{2}{|l|}{ Therapy Pre-CK } \\
\hline Chemotherapy & $12(27.3 \%)$ \\
\hline Targeted therapy & $2(5.1 \%)$ \\
\hline Both & $\mathrm{I}(2.7 \%)$ \\
\hline None & $29(65.9 \%)$ \\
\hline EGFR mutation positive & $19(43 \%)$ \\
\hline \multicolumn{2}{|l|}{ Location of oligo-recurrent lesions } \\
\hline Ipsilateral lung & $26(59.1 \%)$ \\
\hline Contralateral lung & $12(27.3 \%)$ \\
\hline Regional nodes (mediastinum) & $4(9.1 \%)$ \\
\hline Chest wall & $2(4.5 \%)$ \\
\hline \multicolumn{2}{|l|}{ Tumor location } \\
\hline Central & $13(29.5 \%)$ \\
\hline Peripheral & $31(70.5 \%)$ \\
\hline \multicolumn{2}{|l|}{ Number of initial recurrent lesions } \\
\hline I lesion & $4 I(93.2 \%)$ \\
\hline 2-3 lesions & $3(6.8 \%)$ \\
\hline Median tumor size, cm (range) & $2.83 \mathrm{~cm}(1.9$ to $8.1 \mathrm{~cm})$ \\
\hline$<3 \mathrm{~cm}$ & $21(47.7 \%)$ \\
\hline $3-7 \mathrm{~cm}$ & $20(45.5 \%)$ \\
\hline$>7 \mathrm{~cm}$ & $3(6.8 \%)$ \\
\hline \multicolumn{2}{|l|}{ ECOG score Pre-CK } \\
\hline 0 & $26(59.1 \%)$ \\
\hline I & $18(40.9 \%)$ \\
\hline Hemoglobin Levels, median (min, max) & $133(90-168)$ \\
\hline WBC counts, median (min, max) & $5.50(3.5-10.6)$ \\
\hline Neutrophil counts, median (min, max) & $3.23(1.82-7.10)$ \\
\hline Lymphocyte counts, median (min, max) & $1.54(0.56-3.24)$ \\
\hline Platelet counts median (min, max) & $185.5(103-475)$ \\
\hline NLR median (min, max) & $1.90(1.11-5.9)$ \\
\hline PLR median (min, max) & I I $8.39(56.78-456.24)$ \\
\hline BED (Gy) Median (range) & 124.8 Gy (60-180 Gy) \\
\hline CCI Median (range) & $9(7-12)$ \\
\hline
\end{tabular}

(Continued) 
Table I (Continued).

\begin{tabular}{|l|l|}
\hline Characteristics & No. (\%) \\
\hline Therapy Post-SBRT & \\
Absent & $8(18.2 \%)$ \\
Present & $36(81.8 \%)$ \\
\hline
\end{tabular}

Abbreviations: CK, CyberKnife radiosurgery; ECOG score, Eastern Cooperation Oncology Group score; SBRT, stereotactic body radiotherapy; BED $_{10}$, biologically effective dose (using the LQ model with the $\alpha / \beta=10$ Gy); NLR, Neutrophil-tolymphocyte ratio; PLR, Platelet-to-lymphocyte ratio; CCl, Charlson comorbidity index, EGFR-TKI, Epidermal growth factor receptor (EGFR) tyrosine kinase inhibitors.

comorbidity index (CCI) were all significantly associated with OS $(\mathrm{p}<0.05)$ (Table 3). Multivariate analysis demonstrated that.a low level of pre-SBRT neutrophil-tolymphocyte ratio (NLR < 2.68) (hazard ratio: $0.13 ; 95 \%$ CI: $0.04-0.48 ; \mathrm{p}=0.002$ ) and low Charlson comorbidity index $(\mathrm{CCI}>9)$ (hazard ratio: 3.97; 95\% CI: 1.46-10.78; $\mathrm{p}=0.007$ ) (Table 4) indicated a better prognosis and longer survival. Figure 3 illustrates the Kaplan-Meier curves according to the neutrophil-to-lymphocyte ratio and Charlson comorbidity index.

\section{Failure Patterns and Adverse Events}

During the study period, 31 (70.5\%) patients experienced disease progression. Among these, the vast majority of which $(20$ [64.5\%]) were distant metastases. The remainder were local recurrence (4 $[12.9 \%])$ or regional recurrence $(7[22.6 \%])$. The most common sites of distant metastases were contralateral lung (9), brain (7), and bone (3), adrenal gland (1).

All patients completed the course of SBRT on schedule. In total, 10 patients $(22.7 \%)$ developed grade $1-2$ adverse events (AEs). The most common AEs were grade
1-2 radiation pneumonitis (3), grade 2 fatigue (6), grade 2 chest wall pain (1). Additionally, 2 patients with central lesions developed radiation pneumonitis in our study. No patients developed grade 3 or greater pulmonary toxicity, including lung fibrosis and pneumonitis.

\section{Discussion}

In many cases of local or locoregional recurrence of NSCLC, complete surgical resection is technically not possible or suitable. For these patients, management of post-recurrence of non-small cell lung cancer remains challenging because of limitations from initial therapy and presence of medical comorbidities that often preclude aggressive therapy. For these reasons, as a relatively minimally invasive definitive treatment approach, SBRT may be better suited to these patients. However, there is a lack of data regarding SBRT of post-surgical thoracic oligorecurrent NSCLC.

As far as we know, only 3 papers regarding the outcomes of SBRT for postoperative of non-small-cell lung cancer have been published so far $^{16-18}$ (Table 5). Takeda et $\mathrm{al}^{16}$ analyzed the outcomes of SBRT in 23 patients with isolated postoperative local recurrence. LC and OS rates were shown to be 94.7 and $86.8 \%$ at 1 year and 84.0 and $76.4 \%$ at 2 years, respectively. Regarding AEs, 3 patients (13.0\%) suffered from radiation pneumonitis grade $\geq 3$. Agolli et $\mathrm{al}^{17}$ retrospectively analyzed 28 high-risk non-small cell lung cancer (NSCLC) patients with post-surgical loco-regional relapse treated with salvage stereotactic body radiotherapy (SBRT). The 2-year overall survival (OS) and disease-free survival (DFS) were $57.5 \%$ and $36.6 \%$, respectively. $1 / 28$ patient experienced grade 3 toxicity.

Table 2 CyberKnife Radiosurgery Treatment

\begin{tabular}{|c|c|c|c|c|c|c|c|c|c|c|}
\hline & Item & $\mathrm{Cl}$ & $\mathrm{nCl}$ & HI & Coverage (\%) & $\begin{array}{c}\text { Prescription } \\
\text { Dose (Gy) }\end{array}$ & $\operatorname{PTV}\left(\mathrm{cm}^{3}\right)$ & Fx & BED $_{10}(G y)$ & Isodose (\%) \\
\hline \multirow[t]{3}{*}{ All patients } & Range & $1.01-3.32$ & $1.15-3.47$ & I.12-1.52 & $67.31-99.68$ & $30-60$ & $3.55-157$ & $2-6$ & $60-180$ & $66-89$ \\
\hline & Mean & 1.28 & 1.4 & 1.25 & 91.28 & 50.73 & 34.20 & 4.1 & 121.5 & 80 \\
\hline & Median & 1.18 & 1.28 & 1.24 & 94.45 & 50 & 14.20 & 4.5 & 124.8 & 80 \\
\hline \multirow[t]{3}{*}{ Central } & Range & $1.01-1.16$ & $1.18-2.05$ & $|| 8-1.4 \mid$. & $67.31-95.61$ & $40-60$ & $4.23-157$ & $3-6$ & $80-133.3$ & $7 \mid-85$ \\
\hline & Mean & 1.19 & 1.41 & 1.26 & 85.63 & 50.15 & 46.12 & 4.77 & 105.3 & 79.5 \\
\hline & Median & 1.66 & 1.29 & 1.25 & 90.68 & 50 & 26.17 & 5 & 100 & 80 \\
\hline \multirow[t]{3}{*}{ Peripheral } & Range & $1.03-3.32$ & I.15-3.47 & $1.12-1.52$ & 70.49-99.68 & $30-60$ & $3.55-142.84$ & $2-5$ & $60-180$ & 66-89 \\
\hline & Mean & 1.31 & 1.41 & 1.24 & 93.45 & 51.77 & 29.21 & 3.77 & 128.27 & 81 \\
\hline & Median & 1.18 & 1.27 & 1.23 & 95.4 & 51 & 12.71 & 3 & 132 & 81 \\
\hline
\end{tabular}

Abbreviations: Coverage: the coverage is volume of the tumor receiving greater than or equal to the prescription dose divided by the total volume of the tumor times 100; $\mathrm{Cl}$, conformity index; $\mathrm{nCl}$, new conformity index; $\mathrm{HI}$, homogeneity index; $\mathrm{Fx}$, fraction. 

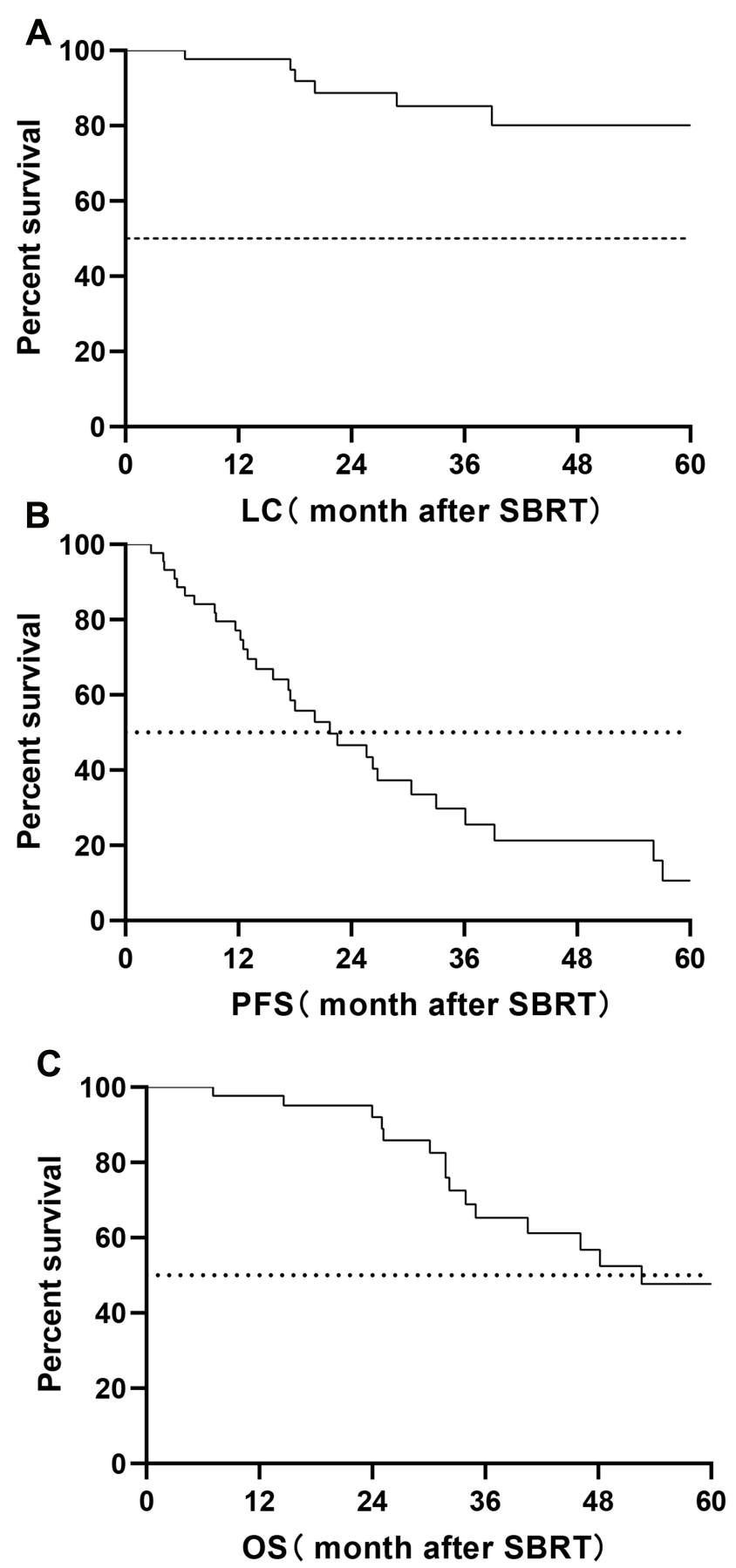

Figure 2 Prognosis of patients underwent slave SBRT. Kaplan-Meier estimates of (A) LC, (B) PFS and (C) OS for patients who underwent SBRT.

Abbreviations: OS, overall survival; PFS, progression-free survival; LC, local control; SBRT, stereotactic body radiotherapy.

Aoki et al $^{18}$ investigated the feasibility of stereotactic body radiotherapy (SBRT) for post-operative oligorecurrence of non-small cell lung cancer. The 1-and 3 -year OS rates were 84.4 and $67.8 \%$, respectively.1-and 3-year PFS rates were 80.8 and $58.7 \%$, respectively. 1-and 3-year LC rate were 97.9 and $94.9 \%$, respectively.
Regarding AEs, 4/52 patients experienced grade 3 or higher adverse events (AEs). In our present study, for the 44 patients with postoperative thoracic oligorecurrence/metastasis, the median follow-up duration after the start of SBRT was 48.5 months $(95 \%$ CI:26.63-70.37 month). The median OS of the 44 patients was 52.6 months (95\% CI:29.59-75.60 months). The 3-year OS, LC, and PFS rates from the start of SBRT were $65.3 \%, 85.1 \%$, and $29.8 \%$, respectively.

Meanwhile, a several of studies have also evaluated the second resection for local recurrence or oligorecurrence in patients who received surgical resection as initial non-small cell lung cancer therapy. ${ }^{19-21}$ Hishida et al $^{19}$ reviewed 28 patients with post-surgical recurrence who received second resection. The median survival time was 25 months, and the 1-, 2-, and 5-year survival rates after recurrence were $89 \%, 59 \%$, and $32 \%$, respectively. Han et $\mathrm{al}^{20}$ compared the prognosis of operative and non-operative treatment for pulmonary oligorecurrence after complete resection of NSCLC. The median post recurrence survival (PRS) was 46.4 months, and the 5-year PRS rates were $67 \%$ in the operative. White et $\mathrm{al}^{21}$ retrospectively reviewed all patients who underwent completion pneumonectomy for recurrent NSCLC. Overall survival at 5 years was $43.1 \%$.

It is worth noting that the prognosis of postoperative thoracic oligo-recurrence is comparable not only to previous studies on SBRT, but also on re-operation for postsurgical recurrence. Additionally, our study revealed that the treatment-related side-effects were well tolerated. This finding is consistent with the results of the previous studies. $^{11,22-24}$

Additionally, we found the pre-SBRT NLR, an inflammatory marker, was closely related to the survival rate. The inflammatory response has been reported to play a critical role in all stages of tumor tumorigenesis, development, and treatment. The level of neutrophils, lymphocytes, and platelets in peripheral blood are significantly correlated to tumor progression. The prognostic values of the NLR and PLR have been applied in non-small cell lung cancer and many other types of cancer. ${ }^{25-28}$ Diem et $\mathrm{al}^{25}$ found increased NLR was associated with worse OS and lower response rates in patients with NSCLC treated with nivolumab. Sebastian et $\mathrm{al}^{29}$ found NLR is associated with inferior outcomes in localized nonsmall cell lung cancer (NSCLC) treated with stereotactic body radiotherapy (SBRT), NLR $>3.6$ was associated with mortality on univariate $(\mathrm{p}=0.010)$ and multivariate 
Table 3 Univariate Analysis for LC, OS

\begin{tabular}{|c|c|c|c|c|}
\hline \multirow[t]{3}{*}{ Variables } & \multicolumn{2}{|l|}{ LC } & \multicolumn{2}{|l|}{ os } \\
\hline & \multicolumn{2}{|c|}{ Univariate } & \multicolumn{2}{|c|}{ Univariate } \\
\hline & HR (95\% Cl) & P-value & HR (95\% Cl) & P-value \\
\hline Sex (female vs male) & $0.58(0.07-4.97)$ & 0.617 & $1.47(0.34-6.38)$ & 0.093 \\
\hline Age at CK $(\geq 74:<74)$ & $3.59(0.72-17.93)$ & 0.119 & $\mathrm{I} .88(0.72-4.91)$ & 0.199 \\
\hline Smoking history (yes vs no) & $1.60(0.32-8.12)$ & 0.571 & $2.15(0.82-5.6)$ & 0.112 \\
\hline Histology (adenocarcinoma vs alternative subtypes) & $1.43(0.26-8.01)$ & 0.678 & $2.15(0.82-5.63)$ & 0.110 \\
\hline $\mathrm{pN}$ classification ( $\mathrm{pN} 0: \mathrm{pN} \geq \mathrm{I}$ ) & $29.13(0.01-1297)$ & 0.432 & $1.63(0.46-0.57)$ & $0.44 I$ \\
\hline Disease-free interval, years ( $\geq 2$ years vs $<2$ years) & $119(0.12-122700)$ & 0.177 & $0.91(0.29-2.83)$ & 0.302 \\
\hline Disease-free interval, years( $\geq$ lyear vs. $<$ lyear) & $31.93(0.01-79136)$ & 0.385 & $1.62(0.64-4.1 I)$ & 0.869 \\
\hline Symptoms at Recurrence (yes vs no) & $0.96(0.112-8.239)$ & 0.097 & $1.13(0.32-4.01)$ & 0.850 \\
\hline ECOG score Pre-SBRT (0 vs I) & $1.87(0.375-9.29)$ & 0.446 & $3.25(1.22-8.65)$ & 0.013 \\
\hline Hemoglobin Levels $(\geq 110$ vs $<110)$ & $0.79(0.09-6.77)$ & 0.828 & $0.73(0.21-2.60)$ & 0.629 \\
\hline $\mathrm{CCl}(>9$ vs $\leq 9)$ & $4.37(0.78-24.34)$ & 0.093 & $3.97(1.46-10.78)$ & 0.007 \\
\hline NLR (<2.68 vs $\geq 2.68)$ & $5.79(0.95-35.25)$ & 0.056 & $8.64(2.43-30.77)$ & 0.001 \\
\hline PLR $(<117.66$ vs $\geq 117.66)$ & $3.57(0.62-20.54)$ & 0.154 & $2.12(0.82-5.65)$ & 0.111 \\
\hline EGFR-TKI (present vs absent) & $5.74(0.67-49.28)$ & 0.111 & $1.25(0.46-3.36)$ & 0.662 \\
\hline Number of initial recurrent lesions(Ilesion vs $2-3$ lesions) & $1.66(0.19-14.45)$ & 0.647 & $0.73(0.21-2.60)$ & 0.629 \\
\hline \multicolumn{5}{|l|}{ Tumor diameter } \\
\hline$\geq 34 \mathrm{~mm}$ & $1.54(0.27-8.7 I)$ & 0.627 & $2.50(0.27-22.87)$ & 0.012 \\
\hline \multicolumn{5}{|l|}{$<34 \mathrm{~mm}$} \\
\hline Tumor location (central vs peripheral) & $2.06(0.24-17.72)$ & 0.509 & $1.27(0.46-3.48)$ & 0.641 \\
\hline \multicolumn{5}{|l|}{ BED (Gy) } \\
\hline$\geq 100$ vs $<100 \mathrm{~Gy}$ & $26.79(0.03-28130)$ & 0.486 & $0.84(0.24-2.99)$ & 0.790 \\
\hline$\geq 120$ vs $<120 \mathrm{~Gy}$ & I.37(0.25-7.47) & 0.719 & $0.91(0.34-2.45)$ & 0.849 \\
\hline Post-SBRT therapy (absent vs present) & & & $4.13(0.52$ to 32.94$)$ & 0.071 \\
\hline
\end{tabular}

Abbreviations: HR, hazard ratios; CK, CyberKnife radiosurgery; ECOG, Eastern Cooperation Oncology Group score; SBRT, stereotactic body radiotherapy; BEDI0, biologically effective dose (using the LQ model with the $\alpha / \beta=10 \mathrm{~Gy}$ ); pN, pathological N classification; NLR, Neutrophil-to-lymphocyte ratio; PLR, Platelet-to-lymphocyte ratio, $\mathrm{CCl}$, Charlson comorbidity index.

Table 4 Multivariate Analyses for OS After CK

\begin{tabular}{|l|l|l|}
\hline \multirow{2}{*}{ Variables } & Multivariate & P-value \\
\cline { 2 - 3 } & HR (95\% Cl) & P-value \\
\hline $\begin{array}{l}\text { ECOG score Pre-SBRT (0 vs I) } \\
\text { CCI }(\leq 9 \text { vs }>9)\end{array}$ & $2.905(0.852-9.908)$ & 0.088 \\
NLR $(<2.68$ vs $\geq 2.68)$ & $0.281(0.102-0.777)$ & 0.014 \\
\hline $\begin{array}{l}\text { Tumor diameter } \\
\geq 34 \mathrm{~mm} \\
<34 \mathrm{~mm}\end{array}$ & $0.135(0.038-0.473)$ & 0.002 \\
\hline
\end{tabular}

Abbreviations: HR, hazard ratios; CK, CyberKnife radiosurgery; ECOG, Eastern Cooperation Oncology Group score; SBRT, stereotactic body radiotherapy; NLR: Neutrophil-to-lymphocyte ratio; NLR, Neutrophil-to-lymphocyte ratio; CCl, Charlson comorbidity index.

analysis $(\mathrm{p}=0.023)$. While the association between increased NLR and poor outcomes is not fully understood, however, increasing evidence suggests an important role of neutrophils in promoting multiple steps of the metastatic cascade and inhibiting the immune system by suppressing the activity of lymphocytes and T-cell response. $^{23,30-32}$ Together with the studies described above, our study adds to the growing body of literature supporting inflammatory factors as predictors of clinical outcomes in cancerous persons.

Meanwhile, the reason why the treatment-related sideeffects were well tolerated without fatal toxicity in our study may be that we try our best to balance BED with normal tissue damage. The main organs at risk (OARs), such as the heart, esophagus, lungs, spinal cord, proximal tracheobronchial tree, and brachial plexus, were contoured according to the guidelines outlined in the Radiation Therapy Oncology Group (RTOG) 0236 trial. $^{33}$ Chang et $\mathrm{al}^{34}$ also reported it is crucial to balance coverage of the gross tumor to a BED $>100 \mathrm{~Gy}$ with dose constraints to avoid damage to nearby critical structures when using 
A

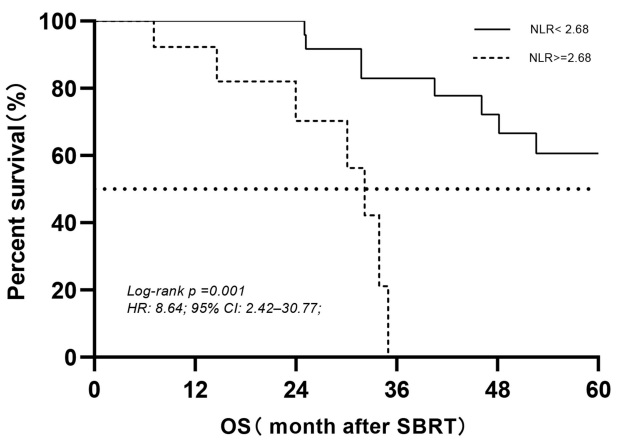

B

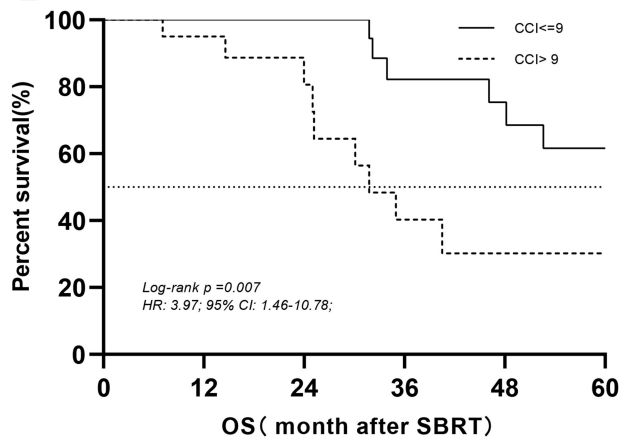

Figure 3 Kaplan-Meier survival curves of overall survival in postsurgical thoracic oligo-recurrence of non-small-cell lung cancer patients. (A) OS according to the NLR (B) OS according to $\mathrm{CCl}$.

Abbreviations: NLR, neutrophil-to-lymphocyte ratio; OS, overall survival; $\mathrm{CCl}$, Charlson comorbidity index.

SABR for treating centrally located tumors. We also found the use of SBRT in a population of post-surgical mediastinal nodal recurrent NSCLC exhibited optimal tolerance and promising local control. The reason may be that BED (median 86.4Gy,80-105.6Gy) is high in these patients, and the addition of other therapy, which may improve local control having a positive impact on survival. Nicosia et al ${ }^{35}$ also found salvage intensity-modulated radiotherapy delivered $(50 \mathrm{~Gy} / 2 \mathrm{~Gy})$ with simultaneous integrated boost $(12.5 \mathrm{~Gy} / 2.5 \mathrm{~Gy})$ is a feasible and well-tolerated treatment option for mediastinal recurrent NSCLC after surgery. Local therapy associated with systemic therapy could be a good option in the treatment of postoperative mediastinal nodal relapse in NSCLC patients unfit to receive a second surgery.

There are several limitations in our study. Primarily, it was conducted at a single institution and using a retrospective design which means some of the intrinsic bias may remain. Additionally, the number of registered patients is limited to 44 subjects, which may have limited the statistical confidence of the results. And due to challenges in confirming the origin of cancer, a few of our recurrent cases may represent secondary lung cancer even though the histologic type is the same.

\section{Conclusion}

The present study confirms the efficacy and safety of SBRT using the CyberKnife for patients with NSCLC displaying post-surgical thoracic oligo-recurrence/metastasis. The low pre-SBRT neutrophil-to-lymphocyte ratio (NLR) and low Charlson comorbidity index were associated with a better prognosis and longer survival and might be considered as reliable and independent prognostic factors in these patients treated with SBRT. To the authors' knowledge, this study is the first study to report the prognostic value of pre-SBRT NLR in patients with post-surgical thoracic recurrence/ metastasis. But the present study is limited by its

Table 5 Study Outcomes of SBRT for the Treatment of Post-Surgical Recurrence of NSCLC

\begin{tabular}{|c|c|c|c|c|c|c|c|}
\hline Study & No & $\begin{array}{l}\text { Median FU } \\
(\mathrm{mo})\end{array}$ & LC (\%) & OS(\%) & PFS(\%) & Dose (Gy/fx) & $\begin{array}{l}\text { Adverse Events } \\
\text { (>Grade2) }\end{array}$ \\
\hline Takeda et al ${ }^{16}$ & 23 & 17 & $\begin{array}{l}\text { ly: } 94.7 \% \\
\text { 2ys: } 86.8 \%\end{array}$ & $\begin{array}{l}\text { ly:84\%; } \\
\text { 2ys:76.4\% }\end{array}$ & $\begin{array}{l}\text { ly:89.5\%; } \\
\text { 2ys:62.5\% }\end{array}$ & $40-60 G y / 5-10 f x$ & 4 patients \\
\hline AGOLLI et al ${ }^{17}$ & 28 & 18 & $\begin{array}{l}\text { ly:96.6\%; } \\
\text { 2ys: } 84.7 \%\end{array}$ & $\begin{array}{l}\text { ly:92.4\%; } \\
\text { 2ys: } 57.5 \%\end{array}$ & $\begin{array}{l}\text { ly:74.4\%; } \\
\text { 2ys: } 36.6 \%\end{array}$ & $23-454 G y / I-3 f x$ & I patient \\
\hline Aoki et $\mathrm{al}^{18}$ & 52 & 25 & $\begin{array}{l}\text { ly:97.9\%; } \\
\text { 3ys: } 94.9 \%\end{array}$ & $\begin{array}{l}\text { ly:84.4\%; } \\
\text { 3ys: } 67.8 \%\end{array}$ & $\begin{array}{l}\text { ly: } 80.8 \% \\
\text { 3ys: } 58.7 \%\end{array}$ & $50-56 G y / 4-10 f x$ & 4 patients \\
\hline Our study & 44 & 48.5 & $\begin{array}{l}\text { ly:97.7\%; } \\
3 y s: 85.1 \% \\
5 y s: 80.1 \%\end{array}$ & $\begin{array}{l}\text { ly:97.7\%; } \\
3 y s: 65.3 \% \\
5 y s: 47.7 \%\end{array}$ & $\begin{array}{l}\text { ly:77.1\%; } \\
\text { 3ys:28.8\%; } \\
\text { 5ys:5.3\%; }\end{array}$ & $30-60 \mathrm{~Gy} / 2-6 \mathrm{fx}$ & none \\
\hline
\end{tabular}

Abbreviations: SBRT, stereotactic body radiotherapy; FU, follow-up; OS, overall survival; LC, local control; PFS, progression free survival; y, year; fx, fraction; mo, month. 
retrospective trait, and the patient characteristics and the optimal treatment plan has not yet been identified. Therefore, further research should be done to further verify the therapeutic effect of SBRT, and to explore the correlation between radiation treatments and the inflammatory response to further optimize treatment strategies.

\section{Acknowledgments}

Wen-Cai Li and Zhen Wang are co-first authors for this study.

\section{Disclosure}

The authors report no conflicts of interest in this work.

\section{References}

1. Uramoto H, Tanaka F. Recurrence after surgery in patients with NSCLC.. Transl Lung Cancer Res. 2014;3(4):4. doi:10.3978/j. issn.2218-6751.2013.12.05

2. Hung -J-J, Jeng W-J, Hsu W-H, Chou T-Y, Huang B-S, Wu Y-C. Predictors of Death, local recurrence, and distant metastasis in completely resected pathological stage-i non-small-cell lung cancer. J Thorac Oncol. 2012;7(7):1115-1123. doi:10.1097/JTO.0b013e31824cbad8

3. Sekihara K, Hishida T, Yoshida J, et al. Long-term survival outcome after postoperative recurrence of non-small-cell lung cancer: who is 'cured' from postoperative recurrence? Eur J Cardiothorac Surg. 2017;52(3):522-528. doi:10.1093/ejcts/ezx127

4. Matsuguma H, Nakahara R, Wakamatsu I, et al. Definitive local therapy for oligo-recurrence in patients with completely resected non-small cell lung cancer. Am J Clin Oncol. 2020;43(3):210-217. doi:10.1097/COC.0000000000000656

5. Ashworth A, Rodrigues G, Boldt G, Palma D. Is there an oligometastatic state in non-small cell lung cancer? A systematic review of the literature. Lung Cancer. 2013;82(2):2. doi:10.1016/j.lungcan.2013.07.026

6. Ashworth AB, Senan S, Palma DA, et al. An individual patient data metaanalysis of outcomes and prognostic factors after treatment of oligometastatic non-small-cell lung cancer. Clin Lung Cancer. 2014;15(5):5. doi:10.1016/j.cllc.2014.04.003

7. Juan O, Popat S. Ablative therapy for oligometastatic non-small cell lung cancer. Clin Lung Cancer. 2017;18(6):6. doi:10.1016/j.cllc.20 17.03.002

8. Petrelli F, Ghidini A, Cabiddu M, et al. Addition of radiotherapy to the primary tumour in oligometastatic NSCLC: a systematic review and meta-analysis.. Lung Cancer. 2018;126:126. doi:10.1016/j. lungcan.2018.11.017

9. Yuan Q, Wang W, Zhang Q, Wang Y, Chi C, Xu XC. $<\mathrm{p}>$ Clinical Features and Prognostic Factor of Thoracic Postoperative OligoRecurrence of Non-Small-Cell Lung Cancer. Cancer Manag Res. 2020;12:1397-1403. doi:10.2147/CMAR.S230579

10. Terada $Y$, Isaka M, Harada $H$, et al. Radiotherapy for local recurrence of non-small-cell lung cancer after lobectomy and lymph node dissection - can local recurrence be radically cured by radiation? Jpn J Clin Oncol. 2020;50(4):425-433. doi:10.1093/jjco/hyz188

11. Milano MT, Kong F-MS, Movsas B. Stereotactic body radiotherapy as salvage treatment for recurrence of non-small cell lung cancer after prior surgery or radiotherapy. Transl Lung Cancer Res. 2018;8 (1):78-87. doi:10.21037/tlcr.2018.08.15

12. Ning MS, Gomez DR, Heymach JV, Swisher SG. Stereotactic ablative body radiation for oligometastatic and oligoprogressive disease Transl Lung Cancer Res. 2018;8(1):97-106. doi:10.21037/tlcr.20 18.09.21
13. Timmerman RD, Paulus R, Pass HI, et al. Stereotactic Body Radiation Therapy for Operable Early-Stage Lung Cancer: findings From the NRG Oncology RTOG 0618 Trial. JAMA Oncol. 2018;4 (9):1263-1266. doi:10.1001/jamaoncol.2018.1251

14. Shen Z-T, Wu X-H, Li B, Zhu -X-X. Clinical outcomes of CyberKnife stereotactic body radiotherapy for peripheral stage I non-small cell lung cancer. Med Oncol. 2015;32(3):55. doi:10.1007/ s12032-015-0506-1

15. Camp RL, Dolled-Filhart M, Rimm DL. X-tile: a new bio-informatics tool for biomarker assessment and outcome-based cut-point optimization. Clin Cancer Res. 2004;10(21):7252-7259. doi:10.1158/1078-0432.CCR-040713

16. Takeda A, Sanuki N, Eriguchi T, et al. Salvage stereotactic ablative irradiation for isolated postsurgical local recurrence of lung cancer. Ann Thorac Surg. 2013;96(5):1776-1782. doi:10.1016/j.athoracsur.2013.06. 014

17. Agolli L, Valeriani M, Carnevale A, et al. Role of salvage stereotactic body radiation therapy in post-surgical loco-regional recurrence in a selected population of non-small cell lung cancer patients. Anticancer Res. 2015;35(3):457.

18. Aoki S, Yamashita H, Takahashi W, et al. Salvage stereotactic body radiotherapy for post-operative oligo-recurrence of non-small cell lung cancer: a single-institution analysis of 59 patients.. Oncol Lett. 2020;19(4):2695-2704. doi:10.3892/ ol.2020.11407

19. Hishida T, Nagai K, Yoshida J, et al. Is surgical resection indicated for a solitary non-small cell lung cancer recurrence? J Thorac Cardiovasc Surg. 2006;131(4):838-842. doi:10.1016/j.jtcvs.2005.11.028

20. Han SJ, Cho S, Yum S, Kim K, Jheon S. Surgical treatment of pulmonary oligorecurrence after curative resection for non-smallcell lung cancer. Interact Cardiovasc Thorac Surg. 2020;30 (1):18-23. doi:10.1093/icvts/ivz221

21. White A, Kucukak S, Lee DN, et al. Completion pneumonectomy is safe and effective in select patients with recurrent non-small cell lung cancer. $J$ Thorac Dis. 2020;12(3):217-222. doi:10.21037/ jtd.2020.01.51

22. Xiong W, Xu Q, Xu Y, et al. Stereotactic body radiation therapy for post-pulmonary lobectomy isolated lung metastasis of thoracic tumor: survival and side effects. BMC Cancer. 2014;14(1):719. doi:10.1186/1471-2407-14-719

23. Sun B, Brooks ED, Komaki R, et al. Long-term outcomes of salvage stereotactic ablative radiotherapy for isolated lung recurrence of nonsmall cell lung cancer: a phase ii clinical trial. $J$ Thorac Oncol. 2017;12 (6):983-992. doi:10.1016/j.jtho.2017.02.018

24. Song IH, Yeom SW, Heo S, et al. Prognostic factors for post-recurrence survival in patients with completely resected Stage I non-small-cell lung cancer. Eur J Cardiothorac Surg. 2014;45 (2):262-267. doi:10.1093/ejcts/ezt333

25. Diem S, Schmid S, Krapf M, et al. Neutrophil-to-Lymphocyte ratio (NLR) and Platelet-to-Lymphocyte ratio (PLR) as prognostic markers in patients with non-small cell lung cancer (NSCLC) treated with nivolumab. Lung Cancer. 2017;111:176-181. doi:10.1016/j. lungcan.2017.07.024

26. Hirahara T, Arigami T, Yanagita S, et al. Combined neutrophil-lymphocyte ratio and platelet-lymphocyte ratio predicts chemotherapy response and prognosis in patients with advanced gastric cancer. BMC Cancer. 2019;19(1):672. doi:10.1186/s12885-019-5903-y

27. Cannon NA, Meyer J, Iyengar P, et al. Neutrophil-Lymphocyte and Platelet-lymphocyte ratios as prognostic factors after stereotactic radiation therapy for early-stage non-small-cell lung cancer. J Thorac Oncol. 2015;10(2):280-285. doi:10.1097/JTO.0000000000000399

28. Guo D, Li M, Chen D, et al. Neutrophil-to-lymphocyte ratio is superior to platelet-to-lymphocyte ratio as a prognostic predictor in advanced non-small-cell lung cancer treated with first-line platinum-based chemotherapy. Future Oncol. 2019;15(6):625-635. doi:10.2217/fon-2018-0667 
29. Sebastian N, Wu T, Bazan J, et al. Pre-treatment neutrophil-lymphocyte ratio is associated with overall mortality in localized non-small cell lung cancer treated with stereotactic body radiotherapy.. Radiother Oncol. 2019;134:151-157. doi:10.1016/j. radonc.2019.01.032

30. Hsu BE, Shen Y, Siegel PM. Neutrophils: orchestrators of the Malignant Phenotype. Front Immunol. 2020;11:1778. doi:10.3389/ fimmu.2020.01778

31. Ethier J-L, Desautels D, Templeton A, Shah PS, Amir E. Prognostic role of neutrophil-to-lymphocyte ratio in breast cancer: a systematic review and meta-analysis. Breast Cancer Res. 2017;19(1):2. doi:10.1186/s13058-016-0794-1

32. Zhao Y, Rahmy S, Liu Z, Zhang C, Lu X. Rational targeting of immunosuppressive neutrophils in cancer. Pharmacol Ther. 2020;212:107556. doi:10.1016/j.pharmthera.2020.107556
33. Timmerman R, Paulus R, Galvin J, et al. Stereotactic body radiation therapy for inoperable early stage lung cancer.. JAMA. 2010;303 (11):11. doi:10.1001/jama.2010.261

34. Chang JY, Li -Q-Q, Xu Q-Y, et al. Stereotactic ablative radiation therapy for centrally located early stage or isolated parenchymal recurrences of non-small cell lung cancer: how to fly in a "no fly zone". Int J Radiat Oncol Biol Phys. 2014;88(5):1120-1128. doi:10.1016/j.ijrobp.2014.01.022

35. Nicosia L, Agolli L, Reverberi C, et al. Salvage radiotherapy with simultaneous integrated boost in non small-cell lung cancer patients with mediastinal relapse after surgery: a pilot study. Radiat Oncol. 2018;13(1):207. doi:10.1186/s13014-018-1155-2

\section{Publish your work in this journal}

Cancer Management and Research is an international, peer-reviewed open access journal focusing on cancer research and the optimal use of preventative and integrated treatment interventions to achieve improved outcomes, enhanced survival and quality of life for the cancer patient.
The manuscript management system is completely online and includes a very quick and fair peer-review system, which is all easy to use. Visit http://www.dovepress.com/testimonials.php to read real quotes from published authors. 